\title{
Primer reporte de Miroculis Edmunds (Ephemeroptera: Leptophlebiidae) para el departamento del Chocó, Colombia
}

\section{The first record of Miroculis Edmunds (Ephemeroptera: Leptophlebiidae) from Chocó, Colombia}

\author{
Zuleyma Mosquera-Murillo*, Karen Everni Córdoba-Aragón* \\ Resumen
}

Se registra por primera vez el género Miroculis Edmunds (Ephemeroptera: Leptophlebiidae) para el departamento del Chocó, Colombia, previamente reportado para los departamentos de Amazonas y Antioquia. El registro se basa en ninfas colectadas en dos quebradas de los municipios de Quibdó y Unión Panamericana, que se hayan depositadas en la Colección Limnológica del Chocó (CLCH-Insec).

Palabras clave: Ephemeroptera, Ninfas, Quebrada.

\begin{abstract}
The genus Miroculis Edmunds (Leptophlebiidae: Ephemeroptera) is record for the first time in the department of Chocó, Colombia. The genus is previously reported for the departments of Amazonas and Antioquia. Registration is based on nymphs collected into two streams of municipalities in Quibdó and Union Panamericana, which have been deposited in the Limnology Collection of Chocó (CLCH-Insec).
\end{abstract}

Keywords: Ephemeroptera, Nymphs, Streams.

La familia Leptophlebiidae es, dentro del orden Ephemeroptera, probablemente la más grande y diversa en países neotropicales (Flowers y De La Rosa 2010), con aproximadamente 40 géneros y 150 especies, todas pertenecientes a la subfamilia Atalophlebiinae (Domínguez et al. 2009). Las ninfas se caracterizan por vivir en aguas corrientes, limpias y bien oxigenadas, adheridas a rocas, troncos, hojas o vegetación sumergida y unas pocas especies se pueden encontrar enterradas en fondos lodosos o arenosos (Domínguez et al. 2006, Roldán y Ramírez 2008).

El género Miroculis (Ephemeroptera: Leptophlebiidae) fue establecido por Edmunds (1963) por la especie Miroculis rossi Edmunds del Perú y actualmente se encuentra integrado por 17 especies descritas, distribuidas desde Trinidad hasta el norte de Argentina (Edmunds 1963, Savage y Peters 1983, Savage 1987, Domínguez 2007, Peters et al. 2008, Surinam, Salles y Lima 2011, Costa y Mariano de 2013, Gama Neto y Hamada 2013). En Colombia, el género ha sido registrado para el departamento de Antioquia por Savage (1987) y más recientemente por Peters et al. (2008) en el Parque Nacional Natural de la Serranía de Chiribiquete en la región Amazónica. En la actualidad el conocimiento al respecto del género es todavía incipiente, porque no existen más registros formalmente publicados del género para otros departamentos de Colombia.

En este trabajo se informa por primera vez la presencia de ninfas del género Miroculis en el departamento del Chocó, en cuerpos de agua de las cuencas de los ríos Atrato y San Juan, como resultado de una revisión de las muestras depositadas en la Colección Limnológica del Chocó (CLCH-Insec), lo que contribuye a ampliar el rango de distribución del género en el país.

Miroculis Edmunds (Figura 1)

Comentarios. Entre los caracteres que diferencian

* Grupo de Limnología, Facultad de Ciencias Naturales, Programa de Biología, Universidad Tecnología del Chocó, Quibdó, Chocó, Colombia. e-mail: zuleymamosquera@gmail.com kaecoar@yahoo.com

Fecha recepción: Octubre 9, $2013 \quad$ Fecha aprobación: Febrero 12, 2015 Editor asociado: González-Maya J. 


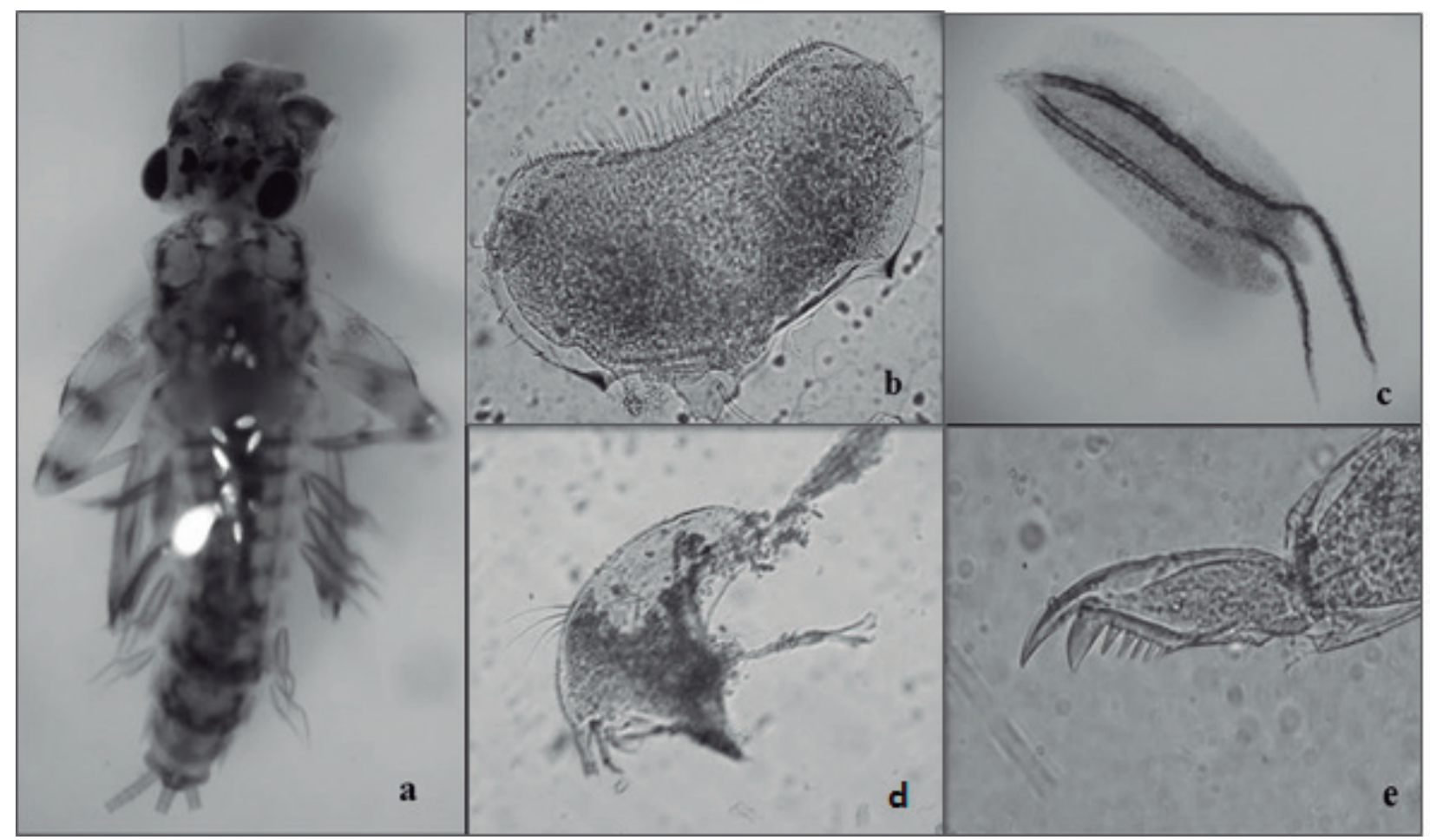

Figura 1. a. Ninfa de Miroculis sp. (hábito en vista dorsal). b. Labro. c. Branquia. d. Mandíbula. e. Uña tarsal.

al género se encuentran: 1) labro más ancho que el clípeo, márgenes laterales redondas formando un ángulo (Figura 1b); 2) márgenes de las mandíbulas con un penacho mediano de setas (Figura 1d); 3) branquias abdominales en los segmentos I-VII, con proyecciones apicales medianas o largas y dos proyecciones posterolaterales más cortas (Figura 1a y 1c); 4) glosa del labro curvada ventralmente; 5) espinas posterolaterales presentes en los segmentos abdominales II o III-IX, más desarrollados en el V-IX; y 6) dentículos en las uñas tarsales progresivamente mayores hacia el ápice; dentículo apical frecuentemente mucho más grande (Figura 1e) (Domínguez et al. 2006). Las ninfas fueron recolectadas mediante red D-net y manualmente con pinzas entomológicas, asociadas con los sustratos vegetación rivereña y piedras en las quebradas Agua Clara (Quibdó) y Sabaletero (Unión Panamericana) respectivamente.

Material examinado: 1 ninfa, COLOMBIA. Chocó. Quibdó. La Troje. Quebrada Agua Clara. 542'45'N 76³6'43”O, 90 m, 16/vii/2013, Torres, H y Perea, M. Cols. [CLCH-Insec]; 1 ninfa, COLOMBIA. Chocó. Unión Panamericana. Salero. Quebrada Sabaletero. 5'19'17.1'” 76³7'38.65.0'W, 20 m, 19/v/2012, Becerra, M. [CLCH-Insec].

\section{Agradecimientos}

Las autoras agradecen al Laboratorio de Limnología, a la Vicerrectoría de Investigaciones y al Centro de Investigación en Biodiversidad y Hábitat (CEIBHA) de la Universidad Tecnológica del Chocó. A la profesora María del Carmen Zúñiga por su colaboración en la confirmación de los especímenes, a la Bióloga Sandra Sánchez Vázquez y a la estudiante de Biología Karen Yuceth Palomeque.

\section{Literatura citada}

Costa SS, Mariano R. 2013. Descripción de una nueva especie de Miroculis Edmunds, 1963 (Ephemeroptera: Leptophlebiidae) de Brasil. Zootaxa. 3599 (5): 495-8.

Domínguez E, Molineri C, Pescador ML, Hubbard MD, Nieto C. 2006. Ephemeroptera of South America. In: Adis J, Arias JR, Rueda Delgado G, Wantzen KM (eds.). Aquatic Biodiversity in Latin America (ALBA). Vol. 2 Sofia: Pensoft; 646 pp.

Domínguez E. 2007. Una nueva especie de Miroculis (Ephemeroptera: Leptophlebiidae) desde el NE de Argentina. Rev Soc Entomol Arg. 66 (3-4): 99-102.

Domínguez E, Molineri C, Nieto C. 2009. Ephemeroptera. En: Domínguez E, Fernández H (eds.). Macroinvertebrados bentónicos sudamericanos: sistemática y biología. San 
Miguel de Tucumán: Fundación Miguel Lillo; 656 pp.

Edmunds GFJr. 1963. A new genus and species of mayfly from Peru (Ephemeroptera: Leptophlebiidae). Pan-Pacific Entomol. 39 (1): 34-6.

Flowers RW, De La Rosa C. 2010. Ephemeroptera. Rev Biol Trop, 58 (Suppl. 4): 63-93.

Gama Neto JL, Hamada N. 2013. Una nueva especie de Miroculis Edmunds, 1963 (Ephemeroptera, Leptophlebiidae) desde el norte de Brasil. Zootaxa. 3734 (5): 597-600.

Peters JG, Domínguez E, Currea-Dereser A. 2008. Species of Miroculis from the Serranía de Chiribiquete in Colombia (Ephemeroptera: Leptophlebiidae: Atalophlebiinae). In: Hauer FR, Stanford JA, Newell RL (eds.). International advances in Ecology, Zoogeography and Systematics of mayflies and stoneflies. 128: 295-305.

Roldán G, Ramírez JJ. 2008. Fundamentos de limnología neotropical. $2^{\mathrm{a}}$ ed. Medellín: Universidad de Antioquia, ACCEFYN, Universidad Católica de Oriente. 442 pp.

Salles FF, Lima MM. 2011. Las nuevas especies y nuevos registros de Miroculis Edmunds (Ephemeroptera: Leptophlebiidae) desde el sureste de Brasil. Zootaxa. 2740: 53-8.

Savage HM, Peters WL. 1983. Systematics of Miroculis and related genera from Northern South America (Ephemeroptera: Leptophlebiidae). Trans Am Entomol Soc. 108: 491-600.

Savage HM. 1987. Two new species of Miroculis from Cerro de la Neblina, Venezuela with new distribution records for Miroculis fittkaui and Microphlebia surinamensis (Ephemeroptera: Leptophlebiidae). Aquatic Insec. 9: 97-108. 